\title{
Decision-Related Activity in Sensory Neurons May Depend on the Columnar Architecture of Cerebral Cortex
}

\author{
Hendrikje Nienborg ${ }^{1,2}$ and Bruce G. Cumming ${ }^{2}$ \\ ${ }^{1}$ Laboratory of Sensorimotor Research, National Eye Institute, National Institutes of Health, Bethesda, Maryland 20892, and ${ }^{2}$ Werner Reichardt Centre for \\ Integrative Neuroscience, University of Tuebingen, 72076 Tuebingen, Germany
}

\begin{abstract}
Many studies have reported correlations between the activity of sensory neurons and animals' judgments in discrimination tasks. Here, we suggest that such neuron-behavior correlations may require a cortical map for the task relevant features. This would explain why studies using discrimination tasks based on disparity in area V1 have not found these correlations: V1 contains no map for disparity. This scheme predicts that activity of V1 neurons correlates with decisions in an orientation-discrimination task. To test this prediction, we trained two macaque monkeys in a coarse orientation discrimination task using band-pass-filtered dynamic noise. The two orientations were always $90^{\circ}$ apart and task difficulty was controlled by varying the orientation bandwidth of the filter. While the trained animals performed this task, we recorded from orientation-selective V1 neurons $(n=82, n=31$ for Monkey $1, n=51$ for Monkey 2$)$. For both monkeys, we observed significant correlation (quantified as "choice probabilities") of the V1 activity with the monkeys' perceptual judgments (mean choice probability $0.54, p=$ $\left.10^{-5}\right)$. In one of these animals, we had previously measured choice probabilities in a disparity discrimination task in V1, which had been at chance $(0.49$, not significantly different from 0.5$)$. The choice probabilities in this monkey for the orientation discrimination task were significantly larger than those for the disparity discrimination task $(p=0.032)$. These results are predicted by our suggestion that choice probabilities are only observed for cortical sensory neurons that are organized in maps for the task-relevant feature.
\end{abstract}

Key words: choice probability; decision; orientation discrimination; striate cortex; V1

\section{Introduction}

For a number of sensory areas and discrimination tasks, trial-totrial correlations between the activity in individual sensory neurons and an animal's perceptual judgment have been observed (Britten et al., 1996; Dodd et al., 2001; Uka and DeAngelis, 2004; Liu and Newsome, 2005; Nienborg and Cumming, 2006; for review, see Nienborg et al., 2012). Although this demonstrates a link between the activity of individual sensory neurons and perception, it also raises new questions. In particular, it is not understood why neurons in some areas show decision-related activity whereas equally selective and informative neurons in other areas do not (Nienborg and Cumming, 2006).

When comparing studies showing decision-related activity in sensory neurons, we noted a common pattern: cortical neurons that displayed decision-related activity were organized in clusters or maps for the stimulus variable that was reported. Decisionrelated activity was found in area MT for direction, speed, and

\footnotetext{
Received June 2, 2013; revised Jan. 17, 2014; accepted Jan. 24, 2014.

Author contributions: H.N. and B.G.C. designed research; H.N. and B.G.C. performed research; H.N. analyzed data; H.N. and B.G.C. wrote the paper.

This work was supported by the Intramural Research Program of the National Institutes of Health-National Eye Institute and a Starting Independent Researcher grant to H.N. from the European Research Council (project acronym: NEUROOPTOGEN). We thank Denise Parker and Beth Nagy for excellent animal care.

The authors declare no competing financial interests.

This article is freely available online through the J Neurosci Author Open Choice option.

Correspondence should be addressed to Hendrikje Nienborg, Werner Reichardt Centre for Integrative Neuroscience, Otfried Mueller Strasse 25, 72076 Tuebingen, Germany. E-mail: hendrikje.nienborg@cin.uni-tuebingen.de.

DOI:10.1523/JNEUROSCI.2340-13.2014

Copyright $\odot 2014$ the authors $\quad 0270-6474 / 14 / 343579-07 \$ 15.00 / 0$
}

disparity discrimination tasks (Britten et al., 1996; Uka and DeAngelis, 2004; Liu and Newsome, 2005) and MT has a maplike organization or at least clustering of neurons with similar tuning for direction (Albright et al., 1984), speed (Liu and Newsome, 2003), and disparity (DeAngelis and Newsome, 1999). An additional study in area MT that found high choice probabilities uses discrimination based on a combination of disparity and direction of motion (Dodd et al., 2001), and clustering for such combinations in the same stimulus has been observed in area MT (K. Krug, B. G. Cumming, and A. J. Parker, unpublished observations). Moreover, areas V2 and IT show clustering for binocular disparity (Ts'o et al., 2001; Yoshiyama et al., 2004) and decision-related activity was observed for disparity discrimination tasks in these areas (Uka et al., 2005; Nienborg and Cumming, 2006, 2007). In contrast, decision-related activity was absent in area V1 for disparity-based discrimination tasks (Grunewald et al., 2002; Nienborg and Cumming, 2006) and clustering for binocular disparity in area V1 is weak if not absent (Prince et al., 2002; Nienborg and Cumming, 2006). One previous study seems at first sight to be at odds with this pattern. A study of neurons of the medial superior temporal area of extrastriate cortex (MST) reported no decision-related activity in a discrimination task for optic-flow patterns (Heuer and Britten, 2004) despite weak clustering for such patterns in MST (Britten and Newsome, 1998). However, this weak clustering for optic flow patterns could be merely a byproduct of the clustering for direction in MST (Celebrini and Newsome, 1994). If this were the case, these data are compatible with the observed pattern in cortex, whereas it 


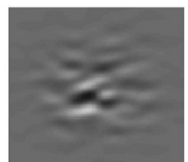

signal: $58 \%$

$0^{\circ}$

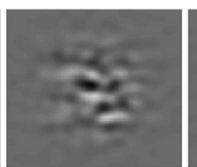

$22 \%$

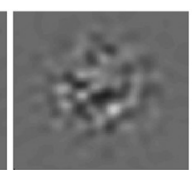

$5 \%$

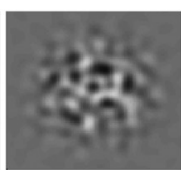

$0 \%$

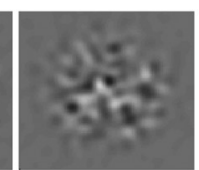

$5 \%$

$90^{\circ}$

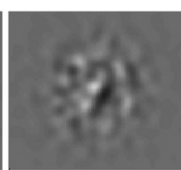

$22 \%$

$90^{\circ}$

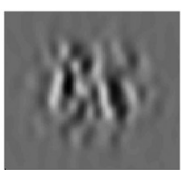

$58 \%$

Figure 1. Stimulus used for coarse orientation discrimination task. Left three panels show example random seeds of the stimulus at $0^{\circ}$ (horizontal) at decreasing signal strength ( $58 \%, 22 \%, 5 \%$ from left to right). Analogous examples for the stimulus at $90^{\circ}$ (vertical) are depicted in the right three panels (increasing signal strength from left to right). Middle, Stimulus at the $0 \%$ orientation signal, for which the orientation is undefined. Stimuli were dynamic and a new random seed presented on each video frame. Note that both monkeys usually achieved close to $100 \%$ correct performance at 22\% signal strength (compare Fig. 2).

may not hold for subcortical areas (Liu et al., 2013a; and see Discussion). Together, these results led us to hypothesize that decisionrelated activity is found only for those neurons that are organized in a map-like way for the task-relevant feature.

Following this hypothesis, we suggest that the lack of decisionrelated activity in V1 during discrimination tasks observed in previous studies (Grunewald et al., 2002; Nienborg and Cumming, 2006) reflects the lack of columnar organization for the relevant features. If this interpretation is correct, it should be possible to demonstrate decision-related activity in V1 during an orientation discrimination task given the well known existence of orientation maps in V1 (Hubel and Wiesel, 1968). Here, we tested this hypothesis by simultaneously recording the activity of orientation-selective V1 neurons while two monkeys performed a coarse orientation discrimination task.

\section{Materials and Methods}

Animals. All procedures were performed in accordance with the US Public Health Service policy on the humane care and use of laboratory animals and all protocols were approved by the National Eye Institute Animal Care and Use Committee. Two male macaque monkeys (Macaca mulatta) were used. Under general anesthesia, both animals were implanted with a head post, a recording chamber over the operculum of V1, and scleral search coils (Judge et al., 1980) as described in detail previously (Cumming and Parker, 1999).

Recording. We recorded extracellular activity from orientation-selective V1 neurons in two macaque monkeys. Eye positions of both eyes were recorded (CNC Engineering). Recording procedures were generally identical to those described previously (Cumming and Parker, 1999; Nienborg and Cumming, 2006) with the exception that the majority of the neurons recorded in Monkey 2 (36/51) were recorded using linear multicontact electrode arrays (eight- or 24-channel U-probes; Plexon). For all recordings, spike waveforms were saved to disk and classified offline using custom software to ensure that the same standards were applied for unit isolation. A variety of parameters were calculated for each spike (e.g., amplitude, width, principal component analysis scores) and waveforms were classified as single units if there was a 2D space in which the single unit formed a cluster that was well separated from the other waveforms.

Stimulus. The stimulus was a dynamic (frame rate $60 \mathrm{~Hz}$ ) version of a stimulus that has been used previously in human psychophysics (Beaudot and Mullen, 2006). Each frame consisted of band-pass-filtered 2D white noise. The filter was an orientation-frequency-separable Gaussian. The peak spatial frequency of the filter was adjusted to approximately match the peak spatial frequency of the recorded neuron and the SD (in spatial frequency) was always half of the peak frequency. The orientation filter was a wrapped Gaussian, the SD of which regulated task difficulty. After applying the filter in the Fourier domain and inverse transforming, a 2D Gaussian window was applied to the image to restrict it to the receptive field. The stimulus was centered on the minimum response field and the size was chosen after measuring an area summation curve (response as a function of Gaussian envelope SD). We selected the smallest Gaussian envelope that elicited a near-maximal neuronal response. For our core dataset ( $n=82$ neurons), the mean peak spatial frequency was 2.02 cycles per degree and the mean envelope SD was $0.53^{\circ}$. For each neuron, we measured the orientation tuning using an orientation narrow band (SD of orientation bandwidth $10^{\circ}$ ) version of the filtered noise stimulus. The animals were required to discriminate two orientations: one close to the neuron's preferred orientation and the other orthogonal to the first. The SD of the orientation filter changed from trial to trial. To facilitate comparison with other tasks, we quantified this as the extent to which the orientation signal was concentrated at one orientation by calculating the vector mean of the filter amplitude. This takes a value of 0 for a completely broadband filter and a value of 1 for a sine wave grating. Typical values for the orientation filter SD were $50^{\circ}, 60^{\circ}, 70^{\circ}$, and infinity, corresponding to concentrations of $22 \%, 11 \%, 5 \%$, and $0 \%$ signal, respectively. Example frames of the stimuli with different signal strength are shown in Figure 1. On the 0\% signal trials, the monkeys were rewarded randomly. For each condition, 1000 images (based on independent noise samples) were precomputed and, on each video frame, a random pick from these 1000 images was drawn. A new set of 1000 images for each condition was generated every day to avoid any possibility that the animals could learn to recognize any particular image. Stimuli were usually presented binocularly at zero disparity. In seven cases in which monocular responses were substantially stronger than binocular responses, the stimulus was presented monocularly to the dominant eye. Stimulus duration was always $2 \mathrm{~s}$.

Task. The two macaque monkeys were trained to perform a coarse orientation-discrimination task. After they acquired fixation, the stimulus appeared for $2 \mathrm{~s}$ followed by 2 choice targets either $3^{\circ}$ above and below or $3^{\circ}$ left and right to the fixation marker. To help the animals generalize the discrimination to different orientations, we used two sets of choice targets. For orientation pairs close to the cardinal axes $\left(\left[-20^{\circ}, 70^{\circ}\right],\left[-10^{\circ}\right.\right.$, $\left.\left.80^{\circ}\right],\left[0^{\circ}, 90^{\circ}\right],\left[10^{\circ}, 100^{\circ}\right],\left[20^{\circ}, 110^{\circ}\right]\right)$, the monkeys indicated their decision with vertical saccades ("vertical target sessions") and for oblique orientation pairs $\left(\left[-60^{\circ}, 30^{\circ}\right],\left[-50^{\circ}, 40^{\circ}\right],\left[-40^{\circ}, 50^{\circ}\right],\left[-30^{\circ}, 60^{\circ}\right]\right)$, they were trained to respond with horizontal saccades ("horizontal target sessions"). If the monkeys made an eye movement to the correct choice target, they received a liquid reward. Trials in which the monkeys did not maintain fixation within $0.5^{\circ}\left(0.8^{\circ}\right.$ for Monkey 2$)$ of the fixation point were aborted.

Computation of choice probability. Analysis of choice probability (CP) was restricted to $0 \%$ signal trials and done as described previously (Britten et al., 1992; Nienborg and Cumming, 2006). We only included neurons in which responses to the highest signal strength used during the task for the two orientations differed by a d' $>1$.2. Of the 255 neurons we recorded, 109 passed this threshold. We also required the following behavioral criteria: the monkeys had to choose each of the two orientations on at least 10 trials for the $0 \%$ signal stimulus and on $<70 \%$ of $0 \%$ signal trials to exclude sessions with excessive bias. In addition, performance for the highest signal condition could not be poorer than $80 \%$ correct. These behavioral criteria were met for $82 / 109$ neurons. The median $\mathrm{d}^{\prime}$ at the maximal signal strength for these 82 neurons was 3.69 and the mean eccentricity was $5.29^{\circ}$ (ranging from $2.6^{\circ}$ to $9.7^{\circ}$ ). Because the stimuli could not be optimized for each individual unit during the multichannel recordings, the median $\mathrm{d}^{\prime}$ for the units recorded with multichannel electrodes in Monkey 2 was 1.83 and the median $\mathrm{d}^{\prime}$ was 3.00 for individually recorded units in this monkey.

Compensating for eye movements. Both animals showed a tendency to make microsaccades toward the end of the $2 \mathrm{~s}$ stimulus presentation, the 
direction of which depended on the upcoming choice saccade. This led to a systematic difference in mean eye position as a function of choice in some cases, which could have affected firing rates. To remove this possibility, we discarded trials with large changes in eye position to eliminate any difference in overall mean eye position. For each recording session, we calculated the mean vector difference in eye position as a function of choice. We then removed trials with a large change in position along this direction until we were left with a set of trials in which the mean difference was $<0.01^{\circ}$. Despite this correction, there was a remaining nonsignificant trend toward a difference in mean eye position as a function of choice toward the end of each trial. For this reason, $\mathrm{CP}$ was calculated using only the trials for which the mean eye position was matched, but also removing the final $300 \mathrm{~ms}$ of each trial to further reduce the possibility that choice-dependent differences in anticipatory microsaccades affected the firing rates.

Neurometric and psychophysical thresholds. We measured the discriminability of the neuronal responses to the two orientations at each signal strength, quantified as the area under the receiver operating curve for the response distributions, as described previously (Britten et al., 1992). Neurometric and psychophysical curves were computed as outlined previously (Britten et al., 1992; Nienborg and Cumming, 2006) and plotted as a function of orientation signal. We then fitted the curves with cumulative Gaussian functions using maximum likelihood and only fits that explained at least $75 \%$ of the variance were used ( $n=69$ for Fig. 3). Thresholds were defined as the SD of the cumulative Gaussians.

\section{Results}

We recorded the extracellular activity of single units in V1 while two macaque monkeys performed a coarse discrimination of two orientations that were always $90^{\circ}$ apart. The stimuli consisted of orientation band-pass-filtered dynamic noise. The orientation bandwidth of the filter determined the strength of the orientation signal and varied from trial to trial (see Materials and Methods) to control the difficulty of the task. Example stimuli for the discrimination of horizontal and vertical stimuli are shown in Figure 1.

\section{Behavioral and neuronal performance for coarse orientation discrimination}

The animals learned to perform this task with high accuracy: the mean psychophysical thresholds (defined at $84 \%$ correct) were $10.9 \%$ for Monkey 1 and $9.5 \%$ for Monkey 2. This implies that, for the $22 \%$ signal stimulus (compare Fig. 1), both monkeys performed, on average, at $>98 \%$ correct. The behavioral results for one example session in each monkey are shown in Figure 2 (top row, gray data). Neurons recorded simultaneously systematically changed their firing rate with signal strength. We defined the preferred orientation in the task as the orientation producing the higher firing rate and we assigned these signal strengths a positive value. At the orientation orthogonal to the preferred (null orientation, negative signal), the response decreased with signal strength such that the mean response increased as a monotonic function of (signed) signal strength (Fig. 2, middle row). We then used the responses to each signal strength to compute neurometric curves (Britten et al., 1992; Fig. 2, top row, black symbols) and compared these with the psychophysical curves. In both examples, the psychophysical threshold was slightly lower than the neuronal threshold. This was typical for the population: on average, the monkeys' psychophysical performance slightly exceeded the neuronal performance (Fig. 3; mean psychophysical threshold $10.1 \%$, mean neuronal threshold $11.9 \%$, mean $\mathrm{N} / \mathrm{P}$ ratio $1.20)$. The mean neuronal thresholds were $14.6 \%$ and $10.3 \%$ (10.0\% for units recorded with multichannel electrodes; $11.1 \%$ for individually recorded single units, no significant difference: $p=0.48, t$ test) signal for Monkeys 1 and 2, respectively. Psychophysical and neuronal thresholds were significantly correlated
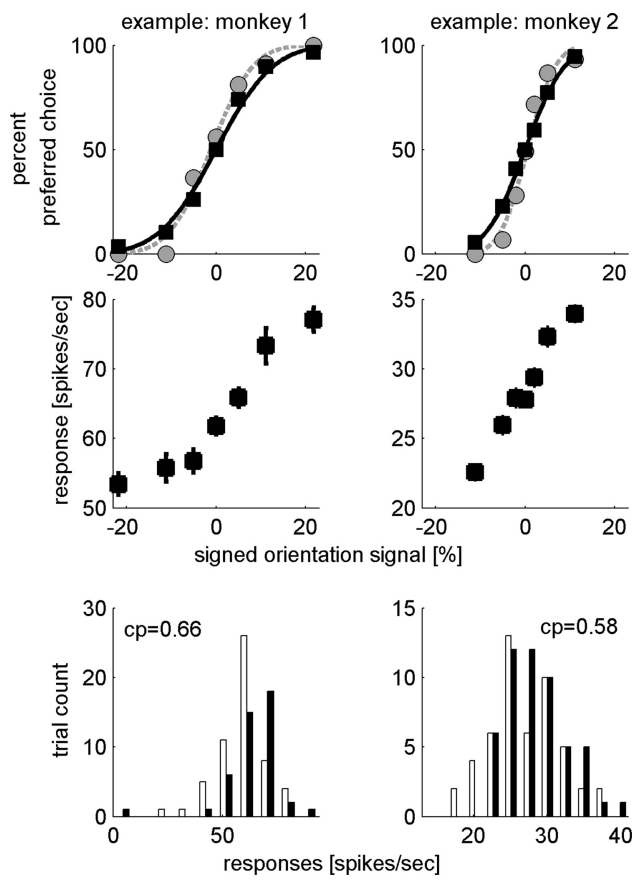

Figure 2. Example neuronal and behavioral responses. Results for one example session are shown for Monkey 1 (left column) and Monkey 2 (right column), respectively. Top, Psychophysical performance (percent choices to the neuron's preferred orientation as a function of signed percent orientation signal, where positive and negative values correspond to the neuron's preferred orientation and that orthogonal to the preferred orientation) are shown by gray circles. Superimposed are the neurometric curves for neurons recorded simultaneously in the same session (black squares). Solid black and dashed gray lines are fits to the neurometric and psychophysical data, respectively. For Monkey 1 and 2, the psychophysical thresholds in these sessions were $7.4 \%$ and $4.8 \%$, respectively, and neuronal thresholds were $10.0 \%(8.5 \%)$ and $6.9 \%(6.4 \%)$, respectively. For consistency with the data on the bottom row, we computed neuronal data for the initial $1.7 \mathrm{~s}$ of each trial. Values in brackets correspond to neuronal thresholds calculated for the entire $2 \mathrm{~s}$ trial. Middle, Mean responses for the same neuron for which the neurometric functions are plotted above are plotted as a function of signed percent orientation signal. Error bars indicate SE. Bottom, Histograms depict the mean response in each trial for which the monkey chose the preferred orientation (black bars) or the null orientation (open bars). For choices to the preferred orientation, the distribution is shifted toward higher response rates, which gives rise to the $C P s>0.5$ for these neurons ( $C P=0.66$ and 0.58 for the example neuron of Monkey 1 and 2, respectively).

( $r=0.34, p=0.0047$, Spearman's rank correlation). However, this correlation was likely largely induced by experimental covariates. We found that both neuronal and psychophysical thresholds were negatively correlated with stimulus size $(r=-0.24, p=$ 0.046 for neuronal threshold, $r=-0.23, p=0.059$ for psychophysical threshold, Spearman's rank correlation) and positively with eccentricity $(r=0.15, p=0.206$ for neuronal threshold and $r=0.12, p=0.311$ for psychophysical threshold, Spearman's rank correlation). Moreover, Monkey 1 had higher neuronal and psychophysical thresholds and there was a weak, nonsignificant trend toward lower thresholds for larger trial numbers $(r=-0.14$, $p=0.254$, for psychophysical threshold and $r=-0.03, p=0.787$ for neuronal threshold, Spearman's rank correlation). The partial correlation between neuronal and psychophysical threshold was not significant $(r=0.24, p>0.05$, Spearman's rank partial correlation) when correcting for the effects of eccentricity, interanimal differences, number of trials, and stimulus size.

Together, the analyses presented here show that both monkeys had learned to perform the orientation discrimination task highly reliably and that the neuronal signals provided by V1 neurons are very suitable to extract the relevant information for this task. 
V1 neurons show decision-related activity in the orientation

discrimination task

Next, we examined trial-by-trial correlations between the activity of individual neurons in V1 and the animal's behavioral decision. We divided the spike counts for each neuron into two groups according to the animal's decision, which resulted in two response distributions. Response distributions for the two example neurons are shown in Figure 2 (bottom row) for trials in which the stimulus always was at $0 \%$ signal. Note that when the animal chose the neuron's preferred orientation, the responses tended to be slightly higher, resulting in a shift in the distribution (Fig. 2, filled bars). This shift suggests that the neuron carried information about the monkey's upcoming perceptual decision.

To quantify this effect, we computed the CP (Britten et al., 1996) for each neuron, which quantifies the probability with which an ideal observer would correctly predict the animal's decision at the end of the trial based on the response of the neuron. Across the population of neurons in both monkeys, the mean CP was 0.54 , significantly larger than chance level (for Fig. $4, n=82, p=10^{-5}$, by resampling). For 17/82 (21\%) of the neurons, CP was significant on an individual neuron level. $\mathrm{CP}$ was also significant in each monkey individually $(0.54, p=0.0014, n=31$ for Monkey 1 and $0.53, p=0.012, n=51$ for Monkey 2; 0.53, $p=0.071, n=36$ for single units recorded with multichannel electrodes, $0.53, p=$ $0.052, n=15$ for individually recorded single units, not statistically different from each other, $p=0.78, t$ test) and the size of mean CP was close to that observed for area MT (mean CP of 0.56) in a direction discrimination task (Britten et al., 1996). Consistent with previous studies in other visual areas (Celebrini and Newsome, 1994; Britten et al., 1996; Parker et al., 2002; Uka and DeAngelis, 2004; Purushothaman and Bradley, 2005; Gu et al., 2008), we found that neurons with lower neuronal thresholds tended to have higher choice probabilities $(r=-0.37, p=$ 0.0020, $n=69$, Spearman's rank correlation; Fig. 3B). However, in our data, this correlation was only significant in Monkey $2(r=$ $-0.42, p=0.0049$, Monkey 2, $n=43, r=-0.30, p=0.14$, Monkey 1, $n=26$, Spearman's rank correlation).

We next performed a number of control analyses to verify that these choice probabilities were not an artifact induced by eye movements.

\section{Effect of eye movements}

Although the monkeys were required to maintain fixation within $0.5^{\circ}\left(0.8^{\circ}\right.$ for Monkey 2$)$ of the fixation marker, systematic differences in eye movements within this window could cause differential firing rates with choice. To verify that such artifacts did not cause the choice probabilities we measured, we analyzed the mean eye position with choice and the effect of microsaccades within the fixation window.

\section{Mean eye position}

To verify that our correction to remove trials to minimize systematic mean differences in eye position as a function of choice was effective, we investigated whether any remaining small, choice-dependent differences in eye position at the end of the trial were systematically associated with CP. We therefore com-
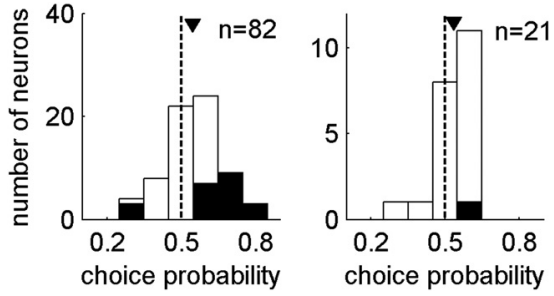

Figure 4. CPs in the population of V1 neurons. Left, Distribution of CPs for all neurons ( $n=$ 82 ) in both monkeys. The mean (filled triangle, 0.54 ) is significantly larger than chance level (dashed vertical line). Right, Distribution of CPs for the subset of neurons $(n=21)$ for which identical $0 \%$ signal stimuli were repeated (two-pass) and for which the animals made different choices on these repeats. The mean (filled triangle, 0.54 ) is significantly larger than chance level (dashed vertical line) and very similar to the mean for all neurons.

puted the correlation between the length of the vector corresponding to the choice-dependent difference in eye position at $1.7 \mathrm{~s}$ after stimulus onset; that is, at the end of the trial component that was used to compute CP. This metric was not significantly correlated with CP $(r=0.01, p=0.92, n=82)$.

\section{Microsaccades}

As described in the Materials and Methods, our analysis of CP was restricted to a subset of trials in which microsaccades produced no difference in mean eye position as a function of choice. However, microsaccades transiently modulate the responses of V1 neurons (Leopold and Logothetis, 1998; Nienborg and Cumming, 2006; Hass and Horwitz, 2011). Spurious choice probabilities due to microsaccades within the fixation window might therefore arise if the frequency, size, or direction of microsaccades systematically differed between the two types of choices and if these differences in microsaccades led to differences in mean firing. To examine this possibility, we identified microsaccades as described previously (Nienborg and Cumming, 2006). We first determined whether there was a significant difference in microsaccade frequency as a function of choice and this was not the case. For sessions in which the animals responded with horizontal saccades, the mean difference was 0.03 microsaccades/trial ( $p=$ $0.48, n=37$ sessions, $t$ test) and, for sessions in which the animals responded with vertical saccades, the mean difference was 0.11 microsaccades/trial ( $p=0.12, n=45$ sessions, $t$ test). Next, we determined whether there was an indication in our neuronal data that differences in microsaccades had contributed to choice 


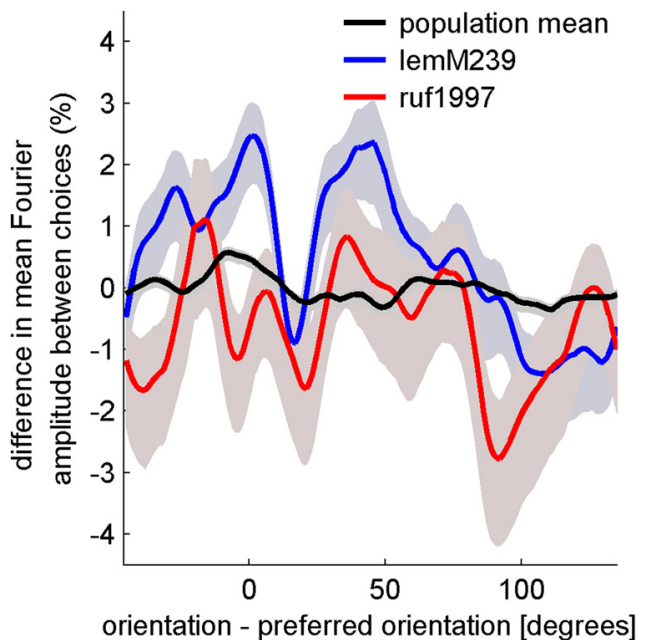

Figure 5. Psychophysical reverse correlation. The ordinate plots the mean difference in Fourier power between images associated with the two choices as a function of orientation relative to the preferred choice. Therefore, an ideal kernel would have a maximum at $0^{\circ}$ and a minimum at $90^{\circ}$. The magnitude is expressed as a percentage of the amplitude difference between two stimuli containing $10 \%$ signal. Data from two example recording sessions are shown \pm SEM. The examples are noisy, but do tend to have larger values near 0 than near 90 . The mean across all sessions is shown by the black solid line \pm SEM. Because of the variation between kernels for different sessions, the mean kernel has a substantially smaller amplitude than the examples.

probabilities. We found no significant correlation between $\mathrm{CP}$ and $\mid$ difference $\mid$ of the saccade frequency with choice $(r=0.05$, $p=0.66, n=82$, Spearman's rank correlation), |difference $\mid$ of saccade size with choice $(r=0.14, p=0.19, n=82$, Spearman's rank correlation), or |differences| in saccade direction as a function of choice $(r=0.07, p=0.53)$.

The above control analyses make it highly unlikely that our observed firing rate differences as a function of choice are due to systematic choice-dependent differences in eye movements.

\section{Stimulus-driven variability}

Because our stimulus with no signal consisted of a random sequence of images, there is always a possibility that random fluctuations in the images caused both changes in neuronal firing rate and in psychophysical reports. If one stimulus happened to have more power in vertical orientations than average, this would both activate neurons that preferred vertical orientations and increase the probability that an animal report a vertical orientation. Our first approach to evaluating this simply calculated the Fourier amplitude spectrum of each image and then calculated the mean power within $\pm 45^{\circ}$ of each choice orientation for each trial. We then quantified the relationship between this variable and the choices with receiver operator characteristic (ROC) analysis (equivalent to the CP calculation). This revealed no significant relationship between stimulus power and choices, with mean ROC value of 0.49 ( $\pm 0.0 .07 \mathrm{SD}, p=0.33$ ).

However, this analysis assumes that the animals place equal weight on power at all stimulus orientations, which may be a poor approximation. To estimate how the animals weighted information in the stimulus, we use psychophysical reverse correlation. For every trial with zero added signal, we calculated the Fourier amplitude for each orientation (summed across spatial frequencies) and then calculated the mean amplitude spectrum for all of the trials associated with each of the two choices. The difference between these means (plotted in Fig. 5) describes how the animals weighted power at different orientations in forming their decisions. Figure 5 shows two examples of these "psychophysical ker- nels." Because each kernel is each derived from a single recording session, they are quite noisy and variable. Nonetheless, they provide the best estimate of how fluctuations in stimulus power at different orientations influenced the choice in a given session. Figure 5 also shows the mean of all the psychophysical kernels, which is smoother but has a smaller amplitude (because of the variation in kernels between sessions).

We used the psychophysical kernel recorded for each session to quantify the effect of stimulus variation on choice. We first multiplied the kernel with the amplitude spectrum of each trial to produce a scalar for each trial that reflects how strongly the orientation content should affect the animals' choices. This "choice predictor" value was significantly related to the animals' choices, with a mean ROC of $0.53( \pm 0.10 \mathrm{SD}, p=0.018)$. Therefore, fluctuations in the stimulus have a modest, but significant, impact on the animals' choices. However, even if spike counts were perfectly correlated with the choice predictor, this would produce a CP of only 0.53 , smaller than the observed mean CP of 0.54 . Nonetheless, to address this possibility directly, we also measured the correlation between spike count and this choice predictor (setting the sign to match each neurons' preferred orientation). The mean value of this correlation was -0.001 ( $\pm 0.10 \mathrm{SD}, p=$ $0.9, t$ test), providing no evidence that fluctuations in the stimulus could contribute to CP. To estimate the effect of both factors combined, we converted the scalar choice predictor into predicted binary choices. For each experiment, they were divided into two groups with a criterion value chosen to match the observed number of choices made by the animal. We then calculated CP for the neuron based on the predicted choices. The mean $\mathrm{CP}$ based on predicted choices was $0.504( \pm 0.07 \mathrm{SD}, p=0.6)$.

One factor that may produce weak correlations between firing rate and the choice predictor is that the shape of the psychophysical kernel does not necessarily match the orientation selectivity of the neuron. We therefore explored separately the extent to which stimulus fluctuations affected spike count (rather than choice). First, we calculated the difference in Fourier power between preferred and null orientations in each trial. This was not significantly correlated with spike count (mean $r=-0.012, p=$ 0.64 ). We also calculated the dot product of the amplitude spectrum for each trial with the orientation tuning curve of each neuron (fit with a von Mises distribution). This value was weakly but not significantly correlated with spike count (mean $r=0.02$, $p=0.07)$. However, this value was not correlated with psychophysical choices: the mean CP for the predicted spike count was 0.50 . Again, this provides no evidence that stimulus fluctuations contributed significantly to the CP that we observed. Note that the weak trend of a correlation between observed and predicted spike count does not imply that stimulus fluctuations have no effect on neuronal firing rates. Rather, when averaged over a $2 \mathrm{~s}$ trial (120 different images), the variance attributable to the stimulus is small relative to other sources of spike count variance.

Finally, in a subset of 21 neurons, we used a "two-pass" stimulus presentation in which each sequence of noise images was shown twice (in separate trials). In approximately half of these pairs, the animal gives different responses on each of the presentations. If CP is calculated using only these trials, then the stimulus ensembles associated with the two choices are identical, so there is no possibility of stimulus-induced CP. Because, for this analysis, CPs were computed on only approximately half of the trials, only one neuron in this subset had an individually statistically significant CP. However, the mean CP in this group was 0.54 (significantly different from $0.5, p=0.014$, Fig. 4 ), similar to the overall mean CP. 


\section{Decision-related activity in V1 for orientation, but not for disparity discrimination}

Our results indicate that, as a population, orientation-selective V1 neurons were informative about the monkeys' perceptual decision in the orientation discrimination task. This contrasts with our previous finding in $\mathrm{V} 1$ for a disparity discrimination task in which we did not observe decision-related activity in disparityselective V1 neurons. Importantly, one of the monkeys performed both tasks. The mean CP in this monkey for the disparity discrimination task was 0.49 (28 neurons, nonsignificant different from 0.5; Nienborg and Cumming, 2006) and the mean choice probabilities between the two tasks in this monkey differed significantly from each other $(p=0.032, t$ test). This suggests that the difference in findings between these two tasks is not a trivial consequence of differences between animals.

\section{Discussion}

In this study, we set out to determine whether V1 neurons show decision-related activity $(\mathrm{CP})$ in a coarse orientation discrimination task. No previous study has reported $\mathrm{CP}$ in $\mathrm{V} 1$ while animals perform a discrimination task. Although this might reflect the early position of V1 in the visual processing hierarchy, we note that previous studies have used tasks for which the relevant feature has no columnar organization in V1. As pointed out in the Introduction, the studies finding CP in extrastriate cortex have used tasks in which the area under study did have a columnar organization for the stimulus feature reported by the animal. Given the columnar organization in V1 for orientation, this study served to test the more general hypothesis that columnar organization is required to observe $\mathrm{CP}$. As predicted by this hypothesis, we find significant choice probabilities in this task in two monkeys.

One reason why a columnar organization may be required is that theoretical (Shadlen et al., 1996) and empirical (Gu et al., 2008; Liu et al., 2013a) studies indicate a close relationship between decision-related activity and noise correlations. Unless a very small number $(<10)$ of sensory neurons is used for a perceptual decision, the observation of choice probabilities requires that neurons that support the same decision are more strongly correlated than neurons supporting opposite decisions in the discrimination task. Noise correlations with this structure (higher within pools than across pools) indicate the presence of some input that is shared by members of a single pool. The origin of this shared input may be feedforward, recurrent, or feedback. In the cortex, anatomical proximity and tuning similarity have consistently been associated with higher noise correlations (Zohary et al., 1994; Kohn and Smith, 2005; Cohen and Newsome, 2008; Smith and Kohn, 2008; Cohen and Maunsell, 2009; Ecker et al., 2010) between neurons. We suggest that the columnar architecture may play a crucial role in generating noise correlations with this pattern. How it is that the columnar architecture might generate these noise correlations will depend on what inputs give rise to the correlation in the first place. If recurrent processing within a cortical area plays an important role, our results suggest that this recurrent processing in some way reflects the columnar architecture (e.g., it might be that recurrent excitatory loops are largely confined within a single column).

We have recently suggested that top-down signals contribute to choice-related activity (Nienborg and Cumming, 2009), which implies they also help to produce this pattern of interneuronal correlations. A columnar architecture may be important for the organization of top-down signals, especially those involved in feature-selective attention. These require that feedback connections influence the activity of a group of neurons that share a certain feature selectivity without affecting other neurons. If neurons with similar selectivities are grouped together, this greatly simplifies the wiring problem: a single feedback connection could modulate a group of similar neurons very straightforwardly. This could dramatically reduce the number of fibers the brain needs to alter the firing of sensory neurons in a context-dependent way and may explain why the brain evolved map-like organization in much of cortex. This principle has implications not only for choice-related activity, but also for the organization of attentional signals. It may only be possible to produce feature-selective attention in neurons that have a columnar organization for the attended feature. Indeed, previous neurophysiological observations of neuronal modulation by feature-selective attention report these for neurons that are organized in clusters for the respective feature (Haenny et al., 1988; Treue and Martinez Trujillo, 1999; McAdams and Maunsell, 2000; Bichot et al., 2005; Cohen and Maunsell, 2011), which is consistent with the scheme put forth here. Columnar architecture may also allow top-down signals to implement "feedback decoding" (Swindale, 2008).

The difficulty of organizing feature-selective feedback connections is not limited to cortical areas. A similar problem may apply to feedback signals to subcortical structures. It has been found recently that neurons in the vestibular nucleus show decision-related activity despite the fact that they are not anatomically clustered by their response preferences (Liu et al., 2013a). This suggests that the scheme we propose does not apply in this case, which may be because the origins of interneuronal correlations in the vestibular nucleus are different from in the cerebral cortex involving complex network interactions (Liu et al., 2013b).

In the cerebral cortex, as we discuss in the Introduction, significant CP (for discrimination tasks) have only been found in areas containing appropriate maps. Because no previous studies of a discrimination task have found significant $\mathrm{CP}$ in area $\mathrm{V} 1$, our results test a strong prediction of the hypothesis: it predicts choice probabilities in area V1, where previous studies have generally not found them. Only one previous study has reported significant choice probabilities in area $\mathrm{V} 1$, for detection of low-contrast Gabors (Palmer et al., 2007). However, decision-related activity in detection tasks can be generated by a process such as alertness, which affects all neurons in an area equally. For this reason, these correlations are often described as "detect probabilities" (Cook and Maunsell, 2002) and the structure of interneuronal correlations required to support these is quite different (Nienborg and Cumming, 2010; Nienborg et al., 2012). Therefore, this result does not test the hypothesis advanced here.

Although we suggest that the columnar architecture plays an important role in generating $\mathrm{CP}$, this does not require that a columnar architecture is always present for a feature where $\mathrm{CP}$ is observed for that feature. If columnar organization at an earlier processing stage is responsible for $\mathrm{CP}$, this will be passed on downstream. For example, even in an extrastriate area that lacked orientation columns, the $\mathrm{CP}$ we describe in V1 should still be evident if that area inherits its orientation selectivity from V1.

The idea that columnar maps improve wiring efficiency has been suggested before (Chklovskii and Koulakov, 2004), but focused on local connections. The enormous advantage for topdown signals results from the fact that these fibers travel further such that reducing their number has a greater impact. Since its discovery (Mountcastle et al., 1955), the role of the cortical columnar structure has been under debate (Horton and Adams, 2005). Our observations suggest an important new function for the columnar architecture of cortex: they greatly simplify the 
organization of top-down feedback that is specific to a neuron's stimulus preferences.

\section{References}

Albright TD, Desimone R, Gross CG (1984) Columnar organization of directionally selective cells in visual area MT of the macaque. J Neurophysiol 51:16-31. Medline

Beaudot WH, Mullen KT (2006) Orientation discrimination in human vision: psychophysics and modeling. Vision Res 46:26-46. CrossRef Medline

Bichot NP, Rossi AF, Desimone R (2005) Parallel and serial neural mechanisms for visual search in macaque area V4. Science 308:529-534. CrossRef Medline

Britten KH, Newsome WT (1998) Tuning bandwidths for near-threshold stimuli in area MT. J Neurophysiol 80:762-770. Medline

Britten KH, Shadlen MN, Newsome WT, Movshon, JA (1992) The analysis of visual motion: a comparison of neuronal and psychophysical performance J Neurosci 12:4745-4765.

Britten KH, Newsome WT, Shadlen MN, Celebrini S, Movshon JA (1996) A relationship between behavioral choice and the visual responses of neurons in macaque MT. Vis Neurosci 13:87-100. CrossRef Medline

Celebrini S, Newsome WT (1994) Neuronal and psychophysical sensitivity to motion signals in extrastriate area MST of the macaque monkey. J Neurosci 14:4109-4124. Medline

Chklovskii DB, Koulakov AA (2004) Maps in the brain: what can we learn from them? Annu Rev Neurosci 27:369-392. CrossRef Medline

Cohen MR, Maunsell JH (2009) Attention improves performance primarily by reducing interneuronal correlations. Nat Neurosci 12:1594-1600. CrossRef Medline

Cohen MR, Maunsell JH (2011) Using neuronal populations to study the mechanisms underlying spatial and feature attention. Neuron 70:11921204. CrossRef Medline

Cohen MR, Newsome WT (2008) Context-dependent changes in functional circuitry in visual area MT. Neuron 60:162-173. CrossRef Medline

Cook EP, Maunsell JH (2002) Dynamics of neuronal responses in macaque MT and VIP during motion detection. Nat Neurosci 5:985-994. CrossRef Medline

Cumming BG, Parker AJ (1999) Binocular neurons in V1 of awake monkeys are selective for absolute, not relative, disparity. J Neurosci 19:5602-5618. Medline

DeAngelis GC, Newsome WT (1999) Organization of disparity-selective neurons in macaque area MT. J Neurosci 19:1398-1415. Medline

Dodd JV, Krug K, Cumming BG, Parker AJ (2001) Perceptually bistable three-dimensional figures evoke high choice probabilities in cortical area MT. J Neurosci 21:4809-4821. Medline

Ecker AS, Berens P, Keliris GA, Bethge M, Logothetis NK, Tolias AS (2010) Decorrelated neuronal firing in cortical microcircuits. Science 327:584587. CrossRef Medline

Grunewald A, Bradley DC, Andersen RA (2002) Neural correlates of structure-from-motion perception in macaque V1 and MT. J Neurosci 22:6195-6207. Medline

Gu Y, Angelaki DE, Deangelis GC (2008) Neural correlates of multisensory cue integration in macaque MSTd. Nat Neurosci 11:1201-1210. CrossRef Medline

Haenny PE, Maunsell JH, Schiller PH (1988) State dependent activity in monkey visual cortex. II. Retinal and extraretinal factors in V4. Exp Brain Res 69:245-259. CrossRef Medline

Hass CA, Horwitz GD (2011) Effects of microsaccades on contrast detection and V1 responses in macaques. J Vis 11:1-17. CrossRef Medline

Heuer HW, Britten KH (2004) Optic flow signals in extrastriate area MST: comparison of perceptual and neuronal sensitivity. J Neurophysiol 91: 1314-1326. CrossRef Medline

Horton JC, Adams DL (2005) The cortical column: a structure without a function. Philos Trans R Soc Lond B Biol Sci 360:837-862. CrossRef Medline

Hubel DH, Wiesel TN (1968) Receptive fields and functional architecture of monkey striate cortex. J Physiol 195:215-243. Medline

Judge SJ, Richmond BJ, Chu FC (1980) Implantation of magnetic search coils for measurement of eye position: an improved method. Vision Res 20:535-538. CrossRef Medline

Kohn A, Smith MA (2005) Stimulus dependence of neuronal correlation in primary visual cortex of the macaque. J Neurosci 25:3661-3673. CrossRef Medline

Leopold DA, Logothetis NK (1998) Microsaccades differentially modulate neural activity in the striate and extrastriate visual cortex. Exp Brain Res 123:341-345. CrossRef Medline

Liu J, Newsome WT (2003) Functional organization of speed tuned neurons in visual area MT. J Neurophysiol 89:246-256. CrossRef Medline

Liu J, Newsome WT (2005) Correlation between speed perception and neural activity in the middle temporal visual area. J Neurosci 25:711-722. CrossRef Medline

Liu S, Dickman JD, Newlands SD, DeAngelis GC, Angelaki DE (2013a) Reduced choice-related activity and correlated noise accompany perceptual deficits following unilateral vestibular lesion. Proc Natl Acad Sci U S A 110:17999-18004. CrossRef Medline

Liu S, Gu Y, DeAngelis GC, Angelaki DE (2013b) Choice-related activity and correlated noise in subcortical vestibular neurons. Nat Neurosci 16: 89-97. CrossRef Medline

McAdams CJ, Maunsell JH (2000) Attention to both space and feature modulates neuronal responses in macaque area V4. J Neurophysiol 83: 1751-1755. Medline

Mountcastle VB, Berman AL, Davies PW (1955) Topographic organization and modality representation in first somatic area of cat's cerebral cortex by method of single unit analysis. Am J Physiol 183:464.

Nienborg H, Cumming B (2010) Correlations between the activity of sensory neurons and behavior: how much do they tell us about a neuron's causality? Curr Opin Neurobiol 20:376-381. CrossRef Medline

Nienborg H, Cumming BG (2006) Macaque V2 neurons, but not V1 neurons, show choice-related activity. J Neurosci 26:9567-9578. CrossRef Medline

Nienborg H, Cumming BG (2007) Psychophysically measured task strategy for disparity discrimination is reflected in V2 neurons. Nat Neurosci 10: 1608-1614. CrossRef Medline

Nienborg H, Cumming BG (2009) Decision-related activity in sensory neurons reflects more than a neuron's causal effect. Nature 459:89-92. CrossRef Medline

Nienborg H, Cohen MR, Cumming BG (2012) Decision-related activity in sensory neurons: correlations among neurons and with behavior. Annu Rev Neurosci 35:463-483. CrossRef Medline

Palmer C, Cheng SY, Seidemann E (2007) Linking neuronal and behavioral performance in a reaction-time visual detection task. J Neurosci 27:81228137. CrossRef Medline

Parker AJ, Krug K, Cumming BG (2002) Neuronal activity and its links with the perception of multi-stable figures. Philos Trans R Soc Lond B Biol Sci 357:1053-1062. CrossRef Medline

Prince SJ, Pointon AD, Cumming BG, Parker AJ (2002) Quantitative analysis of the responses of V1 neurons to horizontal disparity in dynamic random-dot stereograms. J Neurophysiol 87:191-208. Medline

Purushothaman G, Bradley DC (2005) Neural population code for fine perceptual decisions in area MT. Nat Neurosci 8:99-106. CrossRef Medline

Shadlen MN, Britten KH, Newsome WT, Movshon JA (1996) A computational analysis of the relationship between neuronal and behavioral responses to visual motion. J Neurosci 16:1486-1510. Medline

Smith MA, Kohn A (2008) Spatial and temporal scales of neuronal correlation in primary visual cortex. J Neurosci 28:12591-12603. CrossRef Medline

Swindale NV (2008) Feedback decoding of spatially structured population activity in cortical maps. Neural Comput 20:176-204. CrossRef Medline

Treue S, Martinez Trujillo JC (1999) Feature-based attention influences motion processing gain in macaque visual cortex. Nature 399:575-579. CrossRef Medline

Ts'o DY, Roe AW, Gilbert CD (2001) A hierarchy of the functional organization for color, form and disparity in primate visual area V2. Vision Res 41:1333-1349. CrossRef Medline

Uka T, DeAngelis GC (2004) Contribution of area MT to stereoscopic depth perception: choice-related response modulations reflect task strategy. Neuron 42:297-310. CrossRef Medline

Uka T, Tanabe S, Watanabe M, Fujita I (2005) Neural correlates of fine depth discrimination in monkey inferior temporal cortex. J Neurosci 25:10796-10802. CrossRef Medline

Yoshiyama K, Uka T, Tanaka H, Fujita I (2004) Architecture of binocular disparity processing in monkey inferior temporal cortex. Neurosci Res 48:155-167. CrossRef Medline

Zohary E, Shadlen MN, Newsome WT (1994) Correlated neuronal discharge rate and its implications for psychophysical performance. Nature 370:140-143. CrossRef Medline 